\title{
23/25 Gauge Autologous Choroid-RPE Patch Transplantation
}

\author{
Gurkan Erdogan, Abdullah Ozkaya, Irfan Perente \\ Beyoglu Eye Training and Research Hospital, Istanbul, Turkey
}

\begin{abstract}
In this surgical technique report, the advantages of use of vitreous cutters with smaller diameters as vitreoretinal surgical instruments and choroid-RPE patch transplantation that was performed with combination of 23- and 25-gauge vitrectomy systems are described.

Keywords: Choroid; retinal pigment epithelium; vitrectomy.
\end{abstract}

\section{Introduction}

There is currently no cure for age-related macular degeneration (AMD). Anti-vascular endothelial growth factor (anti-VEGF) drugs and photodynamic therapy (PDT) are treatment choices that can reduce the risk and progression of severe vision loss, but do not eliminate the disease.

In advanced stages of AMD, submacular surgery and retinal translocation are surgical choices of treatment to prevent further damage to the retina and to ensure nourishment of the fovea. Patients who experience subretinal hemorrhage or retinal pigment epithelium (RPE) tear, and those who do not respond to anti-VEGF treatment for neovascular AMD are candidates for surgical treatment.

Autologous choroid-RPE patch transplantation has been successfully performed for 2 decades and provides promising functional outcomes in cases of neovascular AMD (I-6). Degenerated and damaged tissue or blood under the macula are removed and replacement subretinal tissues provide contact between fovea and healthy tissue. Full-thickness graft of RPE, Bruch's membrane, and choroid are transplanted from the periphery. This complex surgical technique has high risk for intraoperative complications. Since introduction, it has been performed using 20 -gauge vitectomy techniques. The 20-gauge platform includes a wide variety of multifunctional instruments, available in straight, vertical, and curved designs.

The present study is a description of a technique to take advantage of features of vitreous cutters with smaller diameters and use combination of 23- and 25-gauge vitrectomy systems to perform patch transplantation.

\section{Surgical Technique}

Principal steps of the procedure as previously described for 20 -gauge vitrectomy were performed using 23 -and 25 -gauge instruments on 3 patients (6). All surgeries were performed by single surgeon. Two of the patients underwent phacoemulsification procedure before surgery; the third patient was pseudophakic. Cataract surgery was not performed during the surgical procedures presently described. Three standard 23-gauge sclerotomies were created. Core vitrectomy, posterior hyaloid detachment, peripheral vitrectomy, and vitreous base shaving were performed. Retinal detachment was induced by injecting balanced salt solution into subretinal space through flexible $4 \mathrm{I}$-gauge subretinal cannula (DORC

Address for correspondence: Gurkan Erdogan, MD. Umraniye Training and Research Hospital, Eye Clinic. 


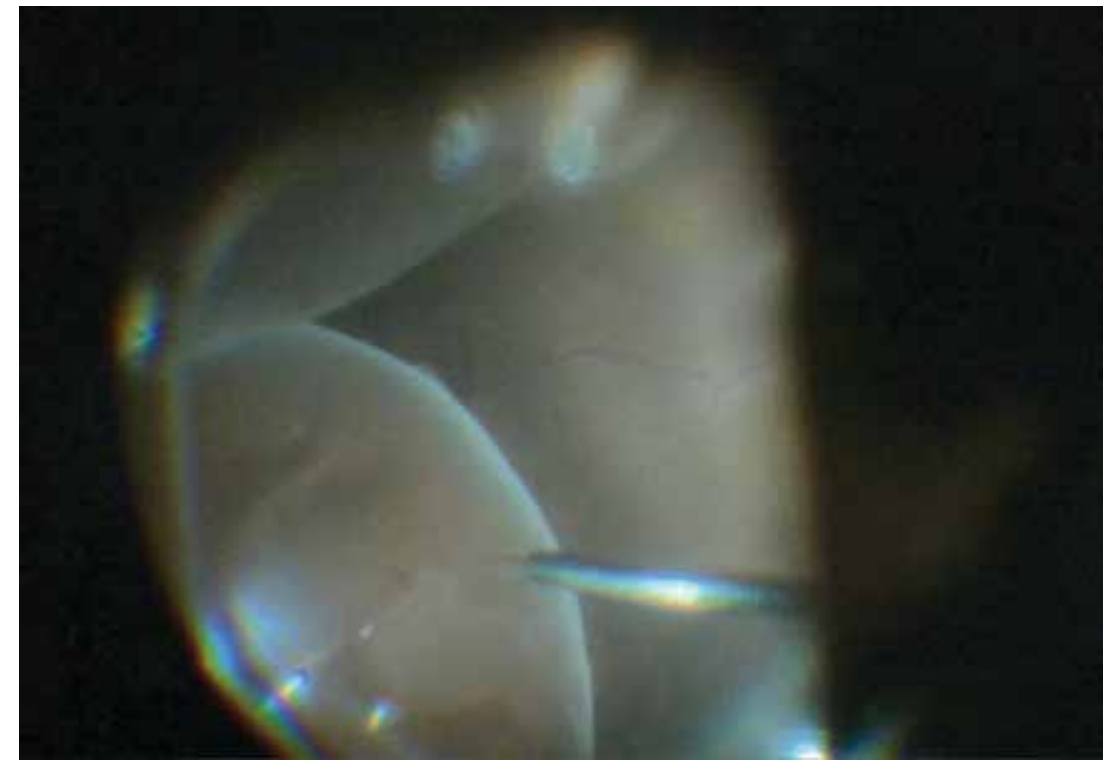

Figure I. Performing retinal detachment with $4 \mathrm{I}$-gauge subretinal cannula.

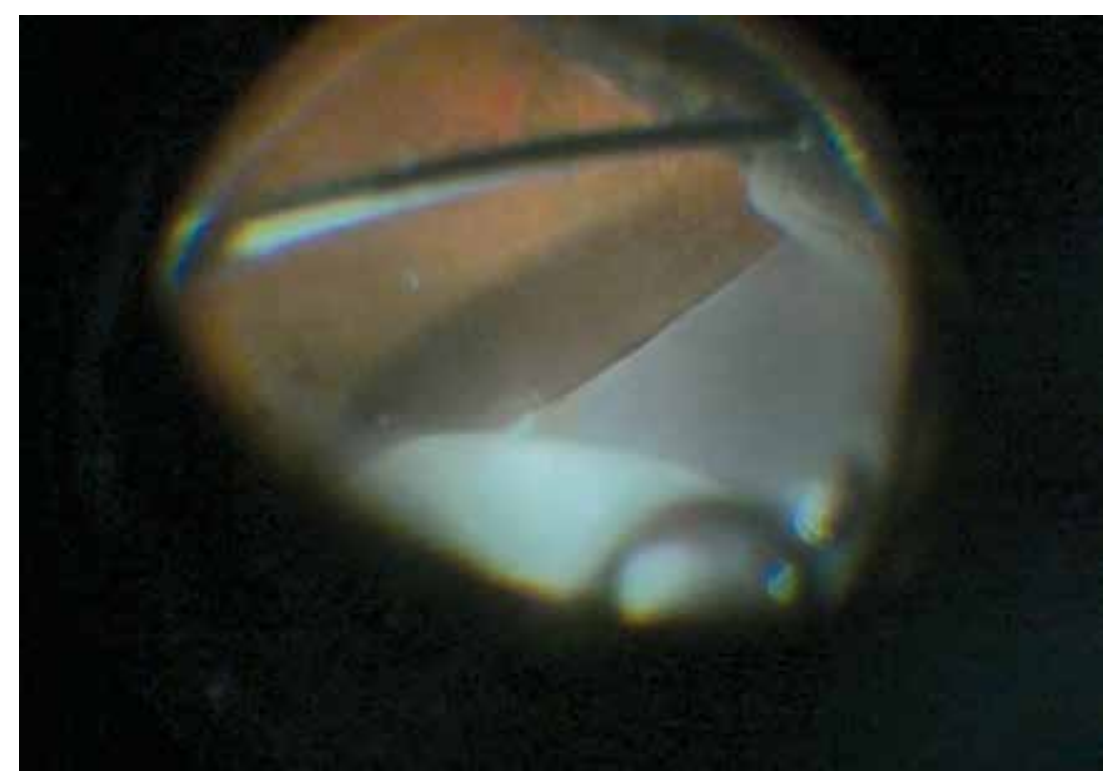

Figure 2. Performing peripheral retinotomy.

Dutch Ophthalmic Research Center International BV, Zuidland, Netherlands) and complete macular detachment was achieved with fluid-air exchange (Figure I). Next, $240^{\circ}$ to $250^{\circ}$ peripheral retinotomy just posterior to the ora serrata was performed with 25-gauge vitreous cutter (Figure 2).

Temporal retina was flapped over nasal side of optic disc and choroidal neovascular membrane was very carefully separated from the retina and underlying choroidal tissue and removed. Bleeding feeder vessels were cauterized on choroidal surface. An area of healthy RPE and choroid was chosen on temporal midperiphery and marked with laser spots or photocoagulation (Figure 3). Approximately $4 \mathrm{~mm}^{2}$ area was incised with vertical scissors at marked borders. Perflu- orocarbon liquid (PFCL) was injected over posterior surface of flapped retina and to level of anterior edge of choroidal patch. Patch was carried by the edges to position under the fovea. PFCL was removed under low pressure and detached retina was repositioned in place, using PFCL once again (Figure 4). Laser retinopexy was performed around the margins of peripheral retinotomy and PFCL-silicone oil exchange was performed.

\section{Results}

Three consecutive patients who underwent autologous choroid-RPE patch transplantation between January and June 2016 were included in this series. Two of the pa- 


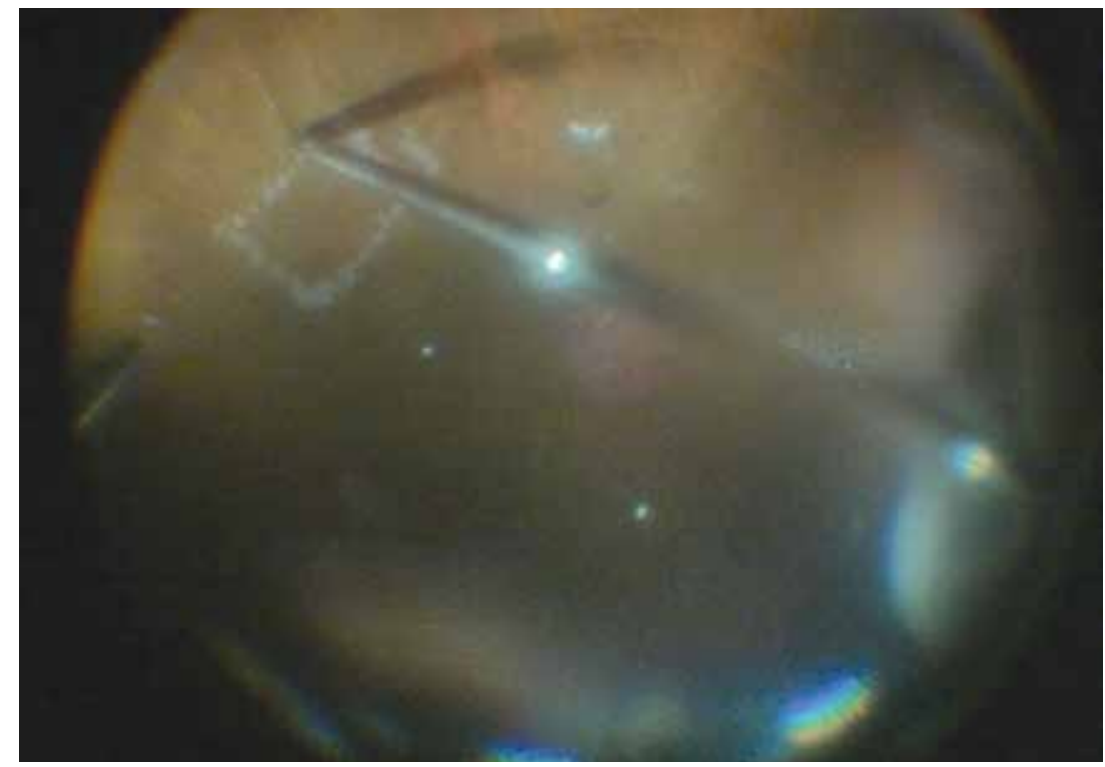

Figure 3. Designation of choroidal patch borders with laser photocoagulation.

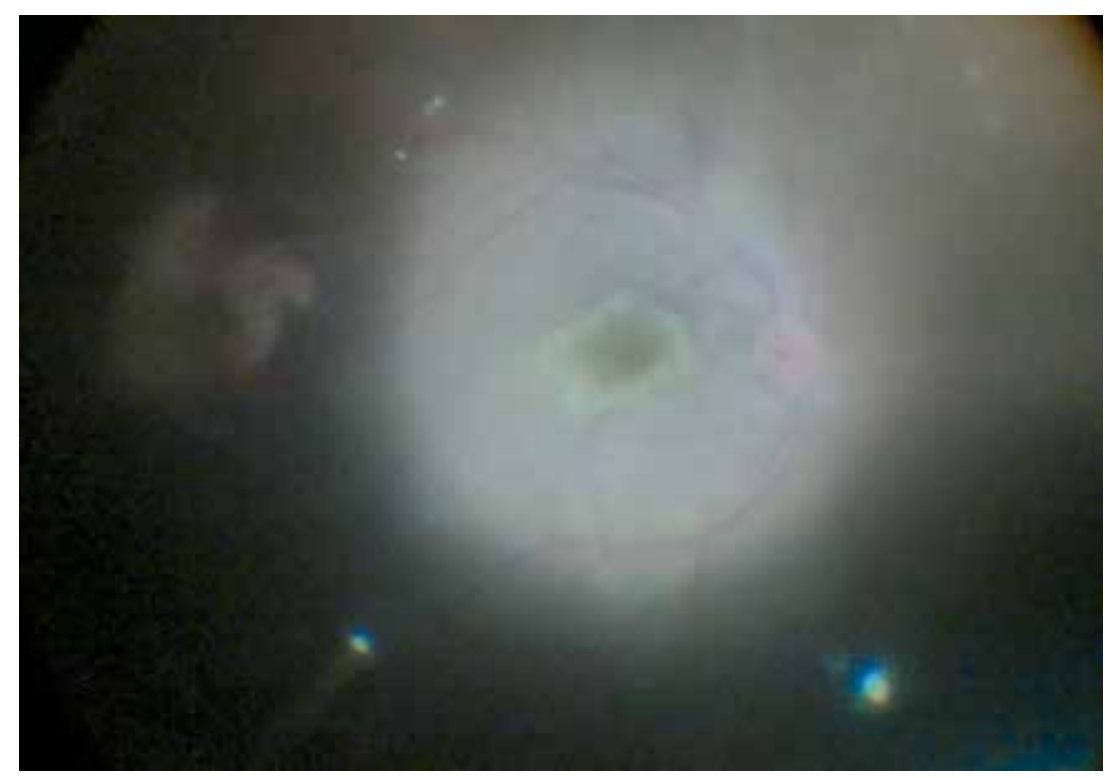

Figure 4. Positioning of choroidal patch under the fovea.

tients were men and I was a woman. Average age was 7I years (range: 68-74 years). Diagnosis of all patients was exudative AMD. One had submacular hemorrhage and 2 were non-responders to anti-VEGF therapy. No complications were observed during the operations or postoperatively over an average follow-up period of 5 months.

\section{Discussion}

Autologous choroidal transplantation is a traumatic, complex, and prolonged surgical procedure. Combination of microincision vitrectomy systems was applied in effort to simplify surgical approach and minimalize surgical trauma of this difficult procedure. Advantages of 23- and 25-gauge vitrectomy systems over 20-gauge include shortened operative time, less inflammation, and reduced patient discomfort; however, there are some disadvantages in terms of variety and rigidity of instruments.

The number of ports was reduced from $4(I, 6)$ to 3 in presently described approach for closed pars plana vitrectomy as no additional port was used for exchange of substances. Peripheral retinotomy was previously performed with 20 -gauge curved scissors. We achieved peripheral retinotomy as close as possible to ora serrata using 25 -gauge vitreous cutter with low aspiration values. Small diameter and short distance between aperture of the vitreous cutter 
and the tip allows the cutter to be used as a multifunctional instrument.

It is the opinion of the authors that microincision vitrectomy systems can be an option in subretinal surgery for advanced AMD cases. Future studies with more patients will add to knowledge about parameters, feasibility, and limitations of this technique.

\section{Disclosures}

Financial Disclosure: This study was not supported by any company. None of the authors has financial or proprietary interests in any material or method mentioned. This data has not been previously published.

Peer-review: Externally peer-reviewed.

Conflict of Interest: None declared.

Authorship Contributions: Involved in design and conduct of the study (GE, AO); preparation and review of the study (GE, AO); data collection (GE); and statistical analysis (not applicable).

\section{References}

I. van Meurs JC, Van Den Biesen PR. Autologous retinal pigment epithelium and choroid translocation in patients with exudative age-related macular degeneration: short-term follow-up. Am J
Ophthalmol 2003; 136:688-95. Crossre

2. Joussen AM, Heussen FM, Joeres S, Llacer H, Prinz B, Rohrschneider K, et al. Autologous translocation of the choroid and retinal pigment epithelium in age-related macular degeneration. Am J Ophthalmol 2006; 142: 17-30. Crossre

3. Treumer F, Bunse A, Klatt C, Roider J. Autologous retinal pigment epithelium-choroid sheet transplantation in age related macular degeneration: morphological and functional results. $\mathrm{Br}$ J Ophthalmol 2007;91:349-53. Crossre

4. MacLaren RE, Uppal GS, Balaggan KS, Tufail A, Munro PM, Milliken $A B$, et al. Autologous transplantation of the retinal pigment epithelium and choroid in the treatment of neovascular age-related macular degeneration. Ophthalmology 2007; I |4:56 I-70,

5. Heussen FM, Fawzy NF, Joeres S, Lux A, Maaijwee K, Meurs JC, et al. Autologous translocation of the choroid and RPE in age-related macular degeneration: I-year follow-up in 30 patients and recommendations for patient selection. Eye (Lond) 2008:799-807.

6. Ma Z, Han L, Wang C, Dou H, Hu Y, Feng X, et al. Autologous transplantation of retinal pigment epithelium-Bruch's membrane complex for hemorrhagic age-related macular degeneration. Invest Ophthalmol Vis Sci 2009;50:2975-8I. Crossre 